\title{
The Algebra of Hurwitz Multizeta Functions
}

\author{
Olivier Bouillot \\ Laboratoire d'informatique Gaspard-Monge (LIGM) \\ Université Paris-Est Marne-la-Vallée \\ Cité Descartes - 5 bd Descartes - 77454 Marne-la-Valle Cedex 2 \\ Received $* * * * *$; accepted after revision +++++ \\ Presented by
}

\begin{abstract}
Multizeta values are real numbers which span a complicated algebra: there exist two different interacting products. A functional analog of these numbers is defined so as to obtain a better understanding of them, the Hurwitz multizeta functions, which span an algebra for which a precise description is wanted. In this note, we prove that the algebra of Hurwitz multizeta functions is a polynomial algebra.
\end{abstract}

\section{Résumé}

Sur l'algèbre des multizêtas de Hurwitz.

Les multizêtas sont des nombres réels possédant une structure d'algèbre complexe : il existe deux produits interagissant. Il est naturel de définir un analogue fonctionnel de ces nombres pour en avoir une meilleure compréhension, ce qui conduit aux multizêtas de Hurwitz, dont on souhaiterait connaître précisémment la structure d'algèbre. Dans cette note, nous montrons que l'algèbre des multizêtas de Hurwitz est une algèbre de polynômes.

Key words: Multizeta values, Hurwitz multizeta functions, Quasi-symmetric functions, Difference equations.

\section{Version française abrégée}

Pour toute séquence $\left(s_{1}, \cdots, s_{r}\right) \in\left(\mathbb{N}^{*}\right)^{r}$, de longueur $r \in \mathbb{N}^{*}$ telle que $s_{1} \geq 2$, on appelle multizêta de Hurwitz (ou encore polyzêta monocentré de Hurwitz) la fonction définie sur $\mathbb{C}-(-\mathbb{N}$ ), par :

$$
\mathcal{H} e^{s_{1}, \cdots, s_{r}}: z \longmapsto \sum_{0<n_{r}<\cdots<n_{1}} \frac{1}{\left(n_{1}+z\right)^{s_{1}} \cdots\left(n_{r}+z\right)^{s_{r}}} .
$$

Email address: olivier.bouillot@univ-mlv.fr (Olivier Bouillot).

URL: http://www-igm.univ-mlv.fr/ bouillot/(Olivier Bouillot). 
La condition $s_{1} \geq 2$ assure la convergence de la série ainsi que le caractère holomorphe de $\mathcal{H} e^{s_{1}, \cdots, s_{r}}$ sur $\mathbb{C}-(-\mathbb{N})$. On définit aussi $\mathcal{H} e^{\emptyset}: z \longmapsto 1$ (Cf. [2] et [3] pour un contexte où ces fonctions apparaissent naturellement en dynamique holomorphe, en lien avec les multitangentes; Cf. [11], [14] et [15] pour d'autres travaux se rapportant à ces fonctions) .

Une telle fonction est une évaluation d'une fonction quasi-symétrique monomiale $M_{s_{1}, \cdots, s_{r}}$ (Cf. [9] pour la première présentation de ces fonctions et [1] pour une présentation plus récente) . Ainsi, cette famille de fonctions engendre une algèbre notée $\mathcal{H} m z f_{c v}$, son produit étant le produit de QSym, appelé aussi bien stuffle, shuffle augmenté, produit de battage contractant, $\cdots$, suivant les auteurs (cf. [8], [7], [3]). L'objectif de cette note est de décrire la structure algébrique de $\mathcal{H} m z f_{c v}$.

On peut remarquer que la famille des multizêtas de Hurwitz est un analogue fonctionnel de celle des multizêtas,ces derniers étant définis par $\mathcal{Z} e^{s_{1}, \cdots, s_{r}}=\mathcal{H} e^{s_{1}, \cdots, s_{r}}(0)$ (Cf. [6], [17] et [18] pour une introduction aux multizêtas). On sait peu de choses sur la nature arithmétique des multizêtas, malgrés quelques avancées récentes (Cf. [4], [5] , [12] et [13]). Ces nombres engendrent aussi une algèbre notée $\mathcal{M} z v_{c v}$ et l'on peut demander une description algébrique de celle-ci. Par exemple, $\mathcal{M} z v_{c v}$ est-elle une algèbre graduée, et si oui, quelle est la suite des dimensions de ses composantes homogènes?

Déterminer la structure algébrique d'une algèbre non formelle, comme $\mathcal{M} z v_{c v}$ ou $\mathcal{H} m z f_{c v}$, est en général une question difficile car, à priori, d'autres produits peuvent entrer en jeu et complexifier la structure de l'algèbre étudiée. C'est en particulier le cas de $\mathcal{M} z v_{c v}$. Dans le cas des multizêtas de Hurwitz, la situation est en fait incomparablement plus simple, car le stuffle, provenant de l'identification des multizêtas de Hurwitz avec une spécialisation des fonctions quasi-symétriques monomiales, est le seul produit possible.

Dans cette note, nous montrons que $\mathcal{H} m z f_{c v}$ est en fait une algèbre polynomiale. Le point clé est le

Théorème 1 La famille $\left(\mathcal{H} e^{s_{1}, \cdots, s_{r}}\right)_{\substack{s_{1}, \cdots \in s_{r} \in \mathbb{N}^{*} \\ s_{1} \geq 2}}$ est $\mathbb{C}(z)$-linéairement indépendante.

Notons que la référence [10] propose une preuve d'une propriété plus faible, l'indépendance linéaire sur $\mathbb{C}$ des multizêtas de Hurwitz ${ }^{1}$. Les corollaires suivants de ce théorème permettent de répondre à toutes les questions que l'on peut poser concernant les multizêtas d'Hurwitz. Par exemple :

Corollaire 0.1 Notons QSym ${ }_{c v}$ la sous-algèbre de QSym engendrée par les monômes $M_{I}$ où $I=\left(i_{1}, \cdots, i_{r}\right)$ est une composition telle que $i_{1} \geq 2$. Alors :

$$
\mathcal{H} m z f_{c v} \simeq Q S y m_{c v} \simeq \mathbb{Q}\left\langle\mathcal{L} y n\left(y_{1} ; y_{2} ; \cdots\right)-\left\{y_{1}\right\}\right\rangle,
$$

où $\mathcal{L} y n\left(y_{1} ; y_{2} ; \cdots\right)$ représente l'ensemble des mots de Lyndon sur l'alphabet $Y=\left\{y_{1} ; y_{2} ; \cdots\right\}$.

Pour plus d'informations sur les mots de Lyndon, on renvoie le lecteur à [16].

Corollaire 0.2 Toute relation algébrique de $\mathcal{H} m z v_{c v}$ est conséquence de l'évaluation du produit de stuffle.

Corollaire 0.3 Notons $\mathcal{H} m z v_{c v, n}$ l'algèbre des multizêtas de Hurwitz de poids n, c'est-à-dire la sousalgèbre de $\mathcal{H} m z v_{c v}$ engendrée par les multizêtas de Hurwitz $\mathcal{H} e^{s_{1}, \cdots, s_{r}}$ vérifiant $s_{1}+\cdots+s_{r}=n$. Alors :

(i) $\mathcal{H} m z v_{c v}$ est graduée par le poids :

$$
\left\{\begin{array}{l}
\mathcal{H} m z v_{c v}=\bigoplus_{n \in \mathbb{N}} \mathcal{H} m z v_{c v, n} \\
\forall(p, q) \in \mathbb{N}^{2}, \mathcal{H} m z v_{c v, p} \cdot \mathcal{H} m z v_{c v, q} \subset \mathcal{H} m z v_{c v, p+q}
\end{array}\right.
$$

1. Selon le rapporteur, qui nous a signalé cette référence, cette preuve contient quelques erreurs.. 
(ii) $\left\{\begin{array}{l}\operatorname{dim} \mathcal{H} m z f_{c v, 0}=1, \operatorname{dim} \mathcal{H} m z f_{c v, 0}=0 . \\ \operatorname{dim} \mathcal{H} m z f_{c v, n}=2^{n-1} \text { for all } n \geq 2 .\end{array}\right.$

\section{Introduction}

For any sequence $\left(s_{1}, \cdots, s_{r}\right) \in\left(\mathbb{N}^{*}\right)^{r}$, of length $r \in \mathbb{N}^{*}$ such that $s_{1} \geq 2$, we define the Hurwitz multizeta function (or mono-center Hurwitz polyzeta), over $\mathbb{C}-(-\mathbb{N})$, by:

$$
\mathcal{H} e^{s_{1}, \cdots, s_{r}}: z \longmapsto \sum_{0<n_{r}<\cdots<n_{1}} \frac{1}{\left(n_{1}+z\right)^{s_{1}} \cdots\left(n_{r}+z\right)^{s_{r}}} .
$$

The condition $s_{1} \geq 2$ ensures the convergence and, consequently, $\mathcal{H} e^{s_{1}, \cdots, s_{r}}$ is a holomorphic function over $\mathbb{C}-(-\mathbb{N})$. We also define $\mathcal{H} e^{\emptyset}: z \longmapsto 1$. (See. [2] and [3] where these functions appear naturally in connection to holomorphic dynamics and multitangent functions ; see also. [11], [14] and [15] for other articles dealing with these functions) .

Such a function is an evaluation of a monomial quasi-symmetric function $M_{s_{1}, \cdots, s_{r}}$ (See at [9], or [1] for a more recent presentation). Therefore, this family spans an algebra $\mathcal{H} m z f_{c v}$, where the product is the product of QSym, known as the stuffle product (also called augmented shuffle, contracting shuffle, quasi-shuffle, $\cdots$ : see $[8],[7], \cdots)$. The aim of this note is to describe the algebraic structure of $\mathcal{H} m z f_{c v}$.

The familly of Hurwitz multizeta functions is a functional analogue of multizeta values, which are defined by $\mathcal{Z} e^{s_{1}, \cdots, s_{r}}=\mathcal{H} e^{s_{1}, \cdots, s_{r}}(0)$ (See [6], [17] and [18]). Little is known on the arithmetical properties of multizeta values, despite of the recent works [4], [5] , [12] and [13]. These numbers span an algebra denoted by $\mathcal{M} z v_{c v}$. An algebraic description of it may be asked (for instance, if it is a graded algebra, and then a formula for the dimensions of homogeneous components, etc).

Elucidating the algebraic structure of an algebra, like $\mathcal{M} z v_{c v}$ or $\mathcal{H} m z f_{c v}$, is in general a difficult question, since no one can be sure there does not exist a second natural product hidden somewhere inside the algebraic structure itself. This is for instance the case in $\mathcal{M} z v_{c v}$. In the case of Hurwitz multizeta functions, the situation is considerably simpler, because the stuffle product, coming from the identification of the Hurwitz multizeta functions with a specialisation of monomial quasi-symmetric functions, is actually the only product to be taken in account.

In this note, we will explain the reasons which elucidate completely the case of $\mathcal{H} m z f_{c v}$. The key point of the proof, which will be the heart of this note, is the following

Theorem 1.1 The family $\left(\mathcal{H} e^{s_{1}, \cdots, s_{r}}\right)_{\substack{n \in \mathbb{N} \\ s_{1}, \cdots, s_{r} \in \mathbb{N}^{*} \\ s_{1} \geq 2}}$ is $\mathbb{C}(z)$-linearly independent.

Let us notice that [10] proposes a proof of a weaker property, the linear independence over $\mathbb{C}$ of the Hurwitz multizeta functions ${ }^{2}$.

The following corollaries answer all the questions that one would ask for the multizeta values.

Corollary 1.2 Let us denote by QSym $_{c v}$ the subalgebra of QSym spanned by the monomials $M_{I}$ with a composition $I=\left(i_{1}, \cdots, i_{r}\right)$ such that $i_{1} \geq 2$. Then:

$$
\mathcal{H} m z f_{c v} \simeq Q S y m_{c v} \simeq \mathbb{Q}\left\langle\mathcal{L} y n\left(y_{1} ; y_{2} ; \cdots\right)-\left\{y_{1}\right\}\right\rangle,
$$

where $\mathcal{L} y n\left(y_{1} ; y_{2} ; \cdots\right)$ denotes the set of Lyndon words over the alphabet $Y=\left\{y_{1} ; y_{2} ; \cdots\right\}$.

2. According to the referee who pointed out this reference, this proofs contains a few errors... 
For more information about Lyndon words, we refer the reader to [16].

Corollary 1.3 Each algebraic relation in $\mathcal{H} m z v_{c v}$ comes from the expansion of stuffle products.

Corollary 1.4 Let us denote the algebra of Hurwitz multizeta functions of weight $n$ by $\mathcal{H} m z v_{c v, n}$, that is, the subalgebra of $\mathcal{H} m z v_{c v}$ spanned by the Hurwitz multizetas $\mathcal{H} e^{s_{1}, \cdots, s_{r}}$ such that $s_{1}+\cdots+s_{r}=n$. Then:

(i) $\mathcal{H} m z v_{c v}$ is graded by the weight:

$$
\begin{gathered}
\left\{\begin{array}{l}
\mathcal{H} m z v_{c v}=\bigoplus_{n \in \mathbb{N}} \mathcal{H} m z v_{c v, n} . \\
\forall(p, q) \in \mathbb{N}^{2}, \mathcal{H} m z v_{c v, p} \cdot \mathcal{H} m z v_{c v, q} \subset \mathcal{H} m z v_{c v, p+q} .
\end{array}\right. \\
\text { (ii) }\left\{\begin{array}{l}
\operatorname{dim} \mathcal{H} m z f_{c v, 0}=1, \operatorname{dim} \mathcal{H} m z f_{c v, 0}=0 . \\
\operatorname{dim} \mathcal{H} m z f_{c v, n}=2^{n-1} \text { for all } n \geq 2 .
\end{array}\right.
\end{gathered}
$$

\section{A fundamental lemma}

Hurwitz multizeta functions are "translations" of multizeta values. Therefore, it is natural to examine how the shift operator acts on such functions. It turns out that the Hurwitz multizeta functions satisfy a fundamental difference equation.

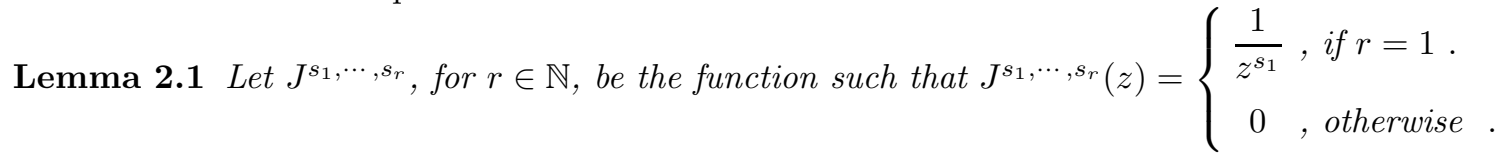

Then, for all $r \in \mathbb{N},\left(s_{1}, \cdots, s_{r}\right) \in\left(\mathbb{N}^{*}\right)^{r}$ such that $s_{1} \geq 2$, we have:

$$
\Delta_{-}\left(\mathcal{H} e^{s_{1}, \cdots, s_{r}}\right)(z)=\frac{1}{z^{s_{r}}} \mathcal{H} e^{s_{1}, \cdots, s_{r-1}}(z) \text {, where } \Delta_{-}(f)(z)=f(z-1)-f(z) .
$$

\section{An example of linear independence of Hurwitz multizeta functions}

As an example, let us consider three rational functions $F_{2}, F_{2,1}$ and $G$ valued in $\mathbb{C}$ such that

$$
F_{2} \mathcal{H} e^{2}+F_{2,1} \mathcal{H} e^{2,1}=G .
$$

If $F_{2,1} \neq 0$, we can assume that $F_{2,1}=1$. Then, applying the operator $\Delta_{-}$to this relation, we obtain:

$$
\widetilde{F}_{2} \mathcal{H} e^{2}=\widetilde{G}
$$

where

$$
\left\{\begin{array}{l}
\widetilde{F}_{2}(z)=\Delta_{-}\left(F_{2}\right)(z)+\frac{1}{z}=\Delta_{-}\left(F_{2}+\mathcal{H} e^{1}\right)(z) \\
\widetilde{G}(z)=\Delta_{-}(G)(z)-\frac{1}{z^{2}} \cdot F_{2}(z-1)
\end{array} \text { and } \mathcal{H} e^{1}(z)=\sum_{n>0}\left(\frac{1}{n+z}-\frac{1}{n}\right) .\right.
$$

From (9), we can easily deduce that $\widetilde{F}_{2}=\widetilde{G}=0$. Consequently, $F_{2}+\mathcal{H} e^{1}$ is a 1-periodic function. But, looking at the possible poles, we can conclude that this is not possible. Therefore, $F_{2,1}=0$, which also implies $F_{2}=G=0$. 
Thus, the function $1, \mathcal{H} e^{2}$ and $\mathcal{H} e^{2,1}$ are $\mathbb{C}(z)$-linearly independent.

This argument will be generalized in the proof of theorem 1.1 and can be enlightened by the following Lemma 3.1 Let $F$ be a rational fontion and $f$ a 1-periodic function.

If, for a $n$-tuple $\left(\lambda_{1}, \cdots, \lambda_{n}\right) \in \mathbb{C}^{n}, F+\sum_{i=1}^{n} \lambda_{i} \mathcal{H} e^{i}=f$ holds, then we necessarily have:

$$
\left\{\begin{array}{l}
\lambda_{1}=\cdots=\lambda_{n}=0 . \\
F \text { and } f \text { are constant functions } .
\end{array}\right.
$$

\section{Linear independence of Hurwitz multizeta functions over the rational function field}

We will give a sketch of proof of Theorem 1.1 based on an induction process. Let us introduce some notations, an order and properties:

$a$. For all $d \in \mathbb{N}$, let $\mathcal{S}_{\leq d}^{\star}$ and $\mathcal{S}_{d}^{\star}$ be the sets defined by $\mathcal{S}_{\leq d}^{\star}=\left\{\underline{\mathbf{s}} \in \mathcal{S}^{\star} ; d^{\circ} \underline{\mathbf{s}} \leq d\right\}$ and $\mathcal{S}_{d}^{\star}=\left\{\underline{\mathbf{s}} \in \mathcal{S}^{\star} ; d^{\circ} \underline{\mathbf{s}}=d\right\}$, where $\mathcal{S}^{\star}=\left\{\left(s_{1}, \cdots, s_{r}\right) \in\left(\mathbb{N}^{*}\right)^{r} ; s_{1} \geq 2\right\}$ and $d^{\circ} \underline{\underline{\mathbf{s}}}=d^{\circ}\left(s_{1}, \cdots, s_{r}\right)=s_{1}+\cdots+s_{r}-r$.

$b$. We can order the sequences of $\mathcal{S}_{d+1}^{\star}$ by numbering first the sequences of length 1 , then those of length 2, etc. For the following proof, let us notice that it will not be necessary to precise the numbering within a length. So, we will consider the sets $\mathcal{S}_{d+1}^{\star}=\left\{\underline{\mathbf{s}}^{n} ; n \in \mathbb{N}^{*}\right\}$ and, for $n \in \mathbb{N}, S_{n}=\left\{\underline{\mathbf{s}}^{i} ; 1 \leq i \leq n\right\}$.

$c$. We will finally consider the following properties:

$$
\begin{aligned}
& \mathcal{D}(d) \text { : " "the family }\left(\mathcal{H} e^{\underline{\mathbf{s}}}\right)_{\underline{\mathbf{s}} \in \mathcal{S}_{\leq d}^{\star}} \text { is } \mathbb{C}(z) \text {-linearly independent." } \\
& \mathcal{P}(d, n) \text { : "the family }\left(\mathcal{H} e^{\underline{\mathbf{s}}}\right)_{\underline{\mathbf{s}} \in \mathcal{S}_{\leq d}^{\star}} \bigcup\left(\mathcal{H} e^{\underline{\mathbf{s}}}\right)_{\underline{\mathbf{s}} \in S_{n}} \text { is } \mathbb{C}(z) \text {-linearly independent." }
\end{aligned}
$$

In order to prove the theorem, we only need to give details on the heredity of the property $\mathcal{D}(n)$. This will also be done by an induction process and the proof boils down to the following implication:

$$
\forall(d, n) \in \mathbb{N}^{2}, \mathcal{P}(d, n) \Longrightarrow \mathcal{P}(d, n+1)
$$

In order to do this, let us assume that Property $\mathcal{P}(d, n)$ holds for a pair $(d, n) \in \mathbb{N}^{2}$ and consider the relation:

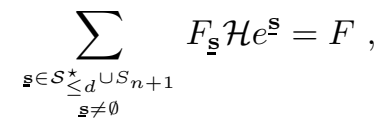

where $F$ and $F_{\underline{\mathbf{s}}}, \underline{\mathbf{s}} \in \mathcal{S}_{\leq d}^{\star} \cup S_{n+1}$, are rational functions. Thanks to $\mathcal{P}(d, n)$, it is sufficient to prove that $F_{\mathbf{s}^{n+1}}=0$. Using a proof by contradiction, we can then assume that $F_{\underline{\mathbf{s}}^{n+1}}=1$.

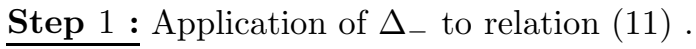

Applying $\Delta_{-}$to (11) and using the induction hypothesis $\mathcal{P}(d, n)$, we obtain

$$
\left\{\begin{array}{l}
\forall \underline{\mathbf{s}} \in\left(\mathcal{S}_{\leq d}^{\star} \cup S_{n}\right)-\{\emptyset\}, \Delta_{-}\left(F_{\underline{\mathbf{s}}}\right)(z)+\sum_{\substack{k \in \mathbb{N}^{*} \\
\mathbf{s} \cdot k \in \mathcal{S}_{\leq d}^{\star} \cup S_{n}}} F_{\underline{\mathbf{s}} \cdot k}(z-1) \cdot J^{k}(z)=0 . \\
\Delta_{-}(F)(z)=\sum_{k=2}^{d+1} F_{k}(z-1) J^{k}(z)+\left(1-\delta_{n, 0}\right) F_{d+2}(z-1) J^{d+2}(z) .
\end{array}\right.
$$


Step 2: A lemma which gives some partial solutions to the system (12) .

Denoting by $\underline{\mathbf{s}}^{n+1}=\underline{\mathbf{u}} \cdot p$ with $p \geq 1$ and $\underline{\mathbf{u}} \in \mathcal{S}_{<d}^{\star} \cup S_{n}$, we can prove that:

Lemma 4.1 Let $r$ be a positive integer and $p \geq 2$.

Let us also consider two $r$-tuples, $\left(n_{1} ; \cdots ; n_{r}\right) \in \mathbb{N}^{r}$ and $\left(k_{1} ; \cdots ; k_{r}\right) \in\left(\mathbb{N}^{*}\right)^{r}$ such that $\sum_{i=1}^{r}\left(k_{i}-1\right) \leq p-2$.

Then, $F_{\underline{\mathbf{u}} \cdot k_{1} \cdot 1^{\left[n_{1}\right]} \ldots k_{r} \cdot 1^{\left[n_{r}\right]}}$ is null if $n_{r}>0$, and is constant if $n_{r}=0$.

Step 3: Revealing the contradiction.

The system (12), applied to $\underline{\mathbf{u}}$, gives us

$$
\Delta_{-}\left(F_{\underline{\mathbf{u}}}+\sum_{k=1}^{p-1} F_{\underline{\mathbf{u}} \cdot k} \mathcal{H} e^{k}+\mathcal{H} e^{p}\right)=0,
$$

where $F_{\underline{\mathbf{u}} \cdot 1}, \cdots, F_{\underline{\mathbf{u}} \cdot p-1}$ are rational fractions which turn out to be constant according to the lemma 4.1 and now denoted by $f_{\underline{\mathbf{u}} \cdot 1}, \cdots, f_{\underline{\mathbf{u}} \cdot p-1}$. Thus, $F_{\underline{\mathbf{u}}}+\sum_{k=1}^{p-1} f_{\underline{\mathbf{u}} \cdot k} \mathcal{H} e^{k}+\mathcal{H} e^{p}$ defines a 1-periodic function. The coefficients being not all zero in this relation, this contradicts Lemma 3.1 and concludes the proof.

\section{References}

[1] F. Bergeron : Algebraic Combinatorics and Coinvariant Spaces, C.M.S. Treatise in Mathematics, C.R.C. Press, 2009, $221 \mathrm{p}$.

[2] O. Boulllot : Invariants analytiques des diffomorphismes et multiztas, Ph.D. thesis, Orsay, (2011), 291p.

[3] O. Boulllot : The Algebra of Multitangent Functions, Journal of Algebra, 116p., 2013, accepted.

[4] F. Brown : Mixed Tate motives over $\mathbb{Z}$, Annals of Math., volume 175 (2012), n2, p. 949-976.

[5] F. BRown : On the decomposition of motivic multiple zeta values, in Galois-Teichmller Theory and Arithmetic Geometry, H. Nakamura et. al. (eds.), Adv. Studies in Pure Math. 68, Math. Soc. Japan, Tokyo, (2012), p. 31-58.

[6] P. CARtier : P. Cartier, Fonctions polylogarithmes, nombres polyzeta et groupes pro-unipotents, Séminaire N. Bourbaki, (2000-2001), exp. 885, p. 137-173.

[7] K. Ebrahimi-Fard, L. Guo : Quasi-shuffles, Mixable Shuffles and Hopf Algebras, J. Algebraic Combinatorics 24, (2006), p. 83-101.

[8] M. Hoffman : Quasi-shuffle products, J. Algebraic Combinatorics. 11, (2000), p. 49-68.

[9] I. Gessel : Multipartite P-partitions and inner products of skew Schur functions, Combinatorics and algebra (Boulder, Colo., 1983), 289?317, Contemp. Math., 34, Amer. Math. Soc., Providence, RI, 1984.

[10] S. Joyner : An algebraic version of the Knizhnik-Zamolodchikov equation, Ramanujan J., 28, no. 3, (2012), p. 361-384.

[11] J.-Y. Enjalbert, H. N. Minh : Combinatorial study of colored Hurwitz polyztas, Discrete Mathematics, 312, 24, (2013), p. 3489-3497.

[12] H. N. Minh : On a conjecture of Pierre Cartier Acta Vietn. Math., 38, n3, (2013), p.339-398.

[13] H. N. Minh : Structure of polyzetas and Lyndon words Vietn. Math. Journ., 41, n4, (2013), p.409-450.

[14] H. N. Minh, G. Jасов, N. E. Oussous, M. Ретітот : De l'algèbre des $\zeta$ de Riemann multivariées à l'algèbre des $\zeta$ de Hurwitz multivariées, journ. electr. sem. Loth. comb, B44e, (2001). 
[15] M. R. Murty, K.Sinha : Multiple Hurwitz zeta function, in Multiple Dirichlet series, automorphic forms and analytic number theory, Proc. Symp. in Pure Math., 75, (2006), p. 135-156.

[16] C. Reutenauer : Free Lie Algebras, London Math. Soc. Monographs, News series 7, Oxford Sciences Publications, Oxford, 1993.

[17] M. Waldschmidt : Valeurs zêta multiples. Une introduction, Journal de Thorie des Nombres de Bordeaux, 12 (2000), p. $581-592$.

[18] V. V. ZUDILIN : Algebraic relations for multiple zeta values, Russian Mathematical Surveys, 58, 2003, p. 1-29. 